\title{
Decay and persistence of spatial coherence during phonon-assisted relaxation in double quantum dots
}

\author{
Paweł Karwat* and Paweł Machnikowski \\ Department of Theoretical Physics, Wroclaw University of Technology, 50-370 Wroclaw, Poland
}

\begin{abstract}
We present a theoretical study of the evolution of spatial coherence during intraband relaxation between exciton states in a pair of vertically stacked semiconductor quantum dots coupled to acoustic phonons. We show that spatial coherence can be transferred to the ground state even in a system of uncoupled non-identical quantum dots if a particular kind of degeneracy between the interlevel energy splittings is present. The phonon-assisted mechanism of coherence transfer leads to a dependence of the amount of the resulting coherence on the inter-dot distance and temperature. We analyze also the impact of carrier-phonon dynamics on a coupled system, where spatial coherence is present in the delocalized ground state.
\end{abstract}

PACS numbers: 78.67.Hc, 71.38.-k, 03.65.Yz

\section{INTRODUCTION}

The physical properties of systems consisting of two or more semiconductor quantum dots (QDs) are rich and cannot be reduced to single-dot characteristics. Experiments demonstrate that collective emission effects play a role in the optical response of such systems ${ }^{1,2}$, which can be attributed to the formation of delocalized bright and dark states in the presence of inter-dot coupling, even in energetically inhomogeneous structures 3 . Phonon-induced carrier transfer ${ }^{\underline{4}}$ in double quantum dots (DQDs) has been predicted to affect the optical emission by redistributing the occupations between bright and dark states ${ }^{5}$. Even though basic quantum-optical concepts of collective emission or superradiance can be transferred from atomic systems to QDs, the relaxation and dephasing effects induced by the coupling to the lattice environment are specific to QD systems and require an extended treatment, as compared to the theory developed in the atomic context $\underline{6}$.

The experimental evidence of collective effects in the emission from self-assembled QD samples, both closely spaced stacks ${ }^{2}$ as well as natural planar ensembles 1 is particularly interesting, as it suggests that such systems cannot be treated as ensembles of independent emitters. The enhanced, "superradiant" emission from the ground state of a QD ensemble is rather intriguing in view of the huge energy inhomogeneity of such a system (as compared to the emission line width). While the optical effect has been successfully modeled under the assumption that the QDs are coupled not only by the relatively weak long-range dipole interactions but also by short range ones (which might result from a combination of Coulomb couplings and tunneling $)^{7}$, the observed difference ${ }^{1}$ between the time-resolved ensemble emission under quasiresonant excitation (optical transition to higher confined shells, leading to a collective enhancement of emission) and non-resonant excitation (transition to wetting layer or bulk states with no enhancement of emission observed) has not been discussed. Since collective emission relies on constructive interference of emission amplitudes from different atoms or QDs, which is possible due to the formation of coherently delocalized Dicke states, description of the dynamics of spatial coherences in coupled QD systems seems to be crucial for the general understanding of the optical properties of such systems. In particular, since spatial coherence can appear as a result of quasiresonant coherent optical excitation, it is interesting to find out if, and under what conditions, it can be transferred to the ground state manifold, from which the emission takes place.

The principal mechanism of intraband transitions in self-assembled QDs is the carrier-phonon coupling and, indeed, models based on phonon-induced processes successfully reproduce experimental data on carrier relaxation ${ }^{8}$. While in single dots phonon-related effects essentially include relaxation 9 and pure dephasing 10 , in DQDs one deals with a broader variety of processes which is due to the rich structure of carrier states in these systems. Apart from intra-dot relaxation, these processes may include inter-dot phonon-assisted tunneling ${ }^{11-15}$ and Coulomb-mediated transfer ${ }^{16}-18$ of carriers, as well as dephasing of spatial coherence of carrier states delocalized over two dots 19,20 .

In this paper, we study theoretically another phononrelated effect in a DQD structure, which may be of importance for the collective luminescence: the phononassisted transfer of spatial coherence from the excited shells of the DQD system to the ground state manifold (corresponding to the quasi-resonant excitation conditions in an optical experiment). We show that after a coherent excitation of the DQD to a spatially delocalized excited states (which is a reasonable assumption in view of the small DQD size as compared to the relevant wavelength of the laser light), the spatial coherence can indeed be conserved during phonon-assisted relaxation in a system of decoupled QDs provided that the inter-level energy differences in the two dots do not differ considerably. While this effect is captured by a Markovian model of the system dynamics, it is lost in the secular approximation inherent in the most standard Lindblad equation approach. We discuss also the evolution of the spatial 


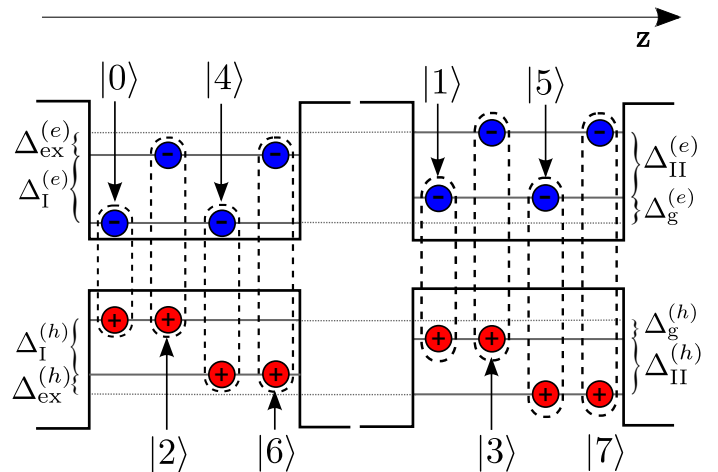

FIG. 1: (Color online) The basis exciton states in the system, $\Delta_{\mathrm{I} / \mathrm{II}}^{(e / h)}$ and $\Delta_{\mathrm{g} / \mathrm{ex}}^{(e / h)}$ are the parameters describing the transition energy mismatch in the dots for electron and the hole, respectively.

coherence in a system of coupled QDs (a quantum dot molecule), where a certain degree of coherent delocalization is built in already in the system ground state and various dynamical scenarios are possible depending on the ratio between the intra-dot and inter-dot relaxation rates.

The paper is organized as follows. In Sec. II we define the model under study. Next, in Sec. III we present the simulations of the evolution of the spatial coherence in DQDs. In particular, Sec. III A deals with uncoupled QDs, while Sec. III B describes coupled system. Sec. IV contains the final discussion and concludes the paper.

\section{MODEL}

The system under study is made up of two large selfassembled and vertically stacked $\mathrm{QDs}^{21}$ occupied by a single exciton, coupled to longitudinal acoustic phonons via the deformation potential. We assume that the differences between the corresponding single-particle levels in the two dots are in the range of a few meV, hence much smaller than the energy of the electron-hole Coulomb attraction. The lowest exciton eigenstates are then formed by spatially direct states, that is, configurations in which the electron-hole pairs reside in the same $\operatorname{dot}^{22,23}$ (presented in Fig. 1). We also assume that the spins of the carriers are fixed. Under these assumptions, the Hilbert space is spanned by eight basis exciton states shown in Fig. 1]. Here the relative energies of the exciton states are parametrized by $\Delta_{\mathrm{I} / \mathrm{II}}^{(e / h)}, \Delta_{\mathrm{g} / \mathrm{ex}}^{(e / h)}$ (see Fig.11). For each particle, three out of the four parameters are independent. For simplicity, we assume that the electronhole Coulomb energy is similar in all the spatially direct configurations.

The total Hamiltonian of the system is

$$
H=H_{\mathrm{DQD}}+H_{\mathrm{ph}}+H_{\mathrm{c}-\mathrm{ph}} \cdot
$$

The first term describes exciton states in the DQD structure and has the form

$$
H_{\mathrm{DQD}}=\sum_{m} \epsilon_{m}|m\rangle\langle m|+\sum_{m \text { even }} V_{m}(|m\rangle\langle m+1|+\text { h.c. }) \text {, }
$$

where $\epsilon_{m}$ are the exciton energies, and the inter-dot couplings are parametrized by $V_{m}$ which is assumed real. We consider the simplest model that is able to account for the effect in question and take into account only a single excited level for each carrier in each dot. We include only couplings between states belonging to the same electron and hole shell, which are energetically close to each other. Even and odd labels correspond to the lower and upper dot, respectively. In the simplest approach, the electron and hole wave functions in the dots are modeled by identical anisotropic Gaussians with identical extensions $l$ in the $x y$ plane and $l_{z}$ along the growth axis $z$ for both particles,

$$
\begin{gathered}
\psi_{\mathrm{g}}(\boldsymbol{r}) \sim \exp \left[-\frac{r_{\perp}^{2}}{2 l^{2}}-\frac{(z \pm D / 2)^{2}}{2 l_{z}^{2}}\right] \\
\psi_{\mathrm{ex}}(\boldsymbol{r}) \sim r_{\perp} \psi_{\mathrm{g}}(\boldsymbol{r}) e^{i \varphi}
\end{gathered}
$$

where $r_{\perp}=\sqrt{x^{2}+y^{2}}$ and $\varphi$ are cylindrical coordinates in the xy plane, the $\mathrm{g} / \mathrm{ex}$ indices refer to the ground and first excited level, and $D$ is the distance between the dots. The exciton wave functions are assumed in the form of products of single-particle functions.

We assume that inter-dot carrier-phonon couplings are negligible due to small overlap of the wave functions confined in different dots. Hence, for the interaction of carriers confined in the DQD with phonons we only retain intra-dot single-particle terms (thus neglecting also the contribution from Coulomb correlations $\left.{ }^{24}\right)$. The excitonphonon coupling is then modeled by the Hamiltonian

$$
\begin{aligned}
H_{\mathrm{c}-\mathrm{ph}} & =\sum_{(m n)}|m\rangle\langle n| \sum_{\boldsymbol{k}} F_{m n}(\boldsymbol{k})\left(b_{\boldsymbol{k}}+b_{-\boldsymbol{k}}^{\dagger}\right) \\
& +\sum_{n}|n\rangle\langle n| \sum_{\boldsymbol{k}} F_{n n}(\boldsymbol{k})\left(b_{\boldsymbol{k}}+b_{-\boldsymbol{k}}^{\dagger}\right)
\end{aligned}
$$

where $(m n)$ denotes summation over pairs of exciton states related by single-particle relaxation within a single QD and $b_{\boldsymbol{k}}, b_{\boldsymbol{k}}^{\dagger}$ are the bosonic operators of the phonon modes. For uncorrelated (product) exciton wave functions, the off-diagonal coupling constants for the exciton states are equal to the electron or hole coupling constant, depending on which particle is involved in the $m \leftrightarrow n$ transition (see Fig. 11), and have the form ${ }^{25}$,

$$
F_{m n}(\boldsymbol{k})=F_{e / h}(\boldsymbol{k}) e^{ \pm i k_{z} D / 2},
$$

where

$$
F_{e / h}(\boldsymbol{k})=\sigma_{\mathrm{e} / \mathrm{h}} \sqrt{\frac{k}{2 \varrho v c_{l}}} i \frac{k_{\perp} l}{2} \exp \left[-\frac{l_{z}^{2} k_{z}^{2}+l^{2} k_{\perp}^{2}}{4}\right] e^{i \phi}
$$


and the "+/-" sign corresponds to the lower and upper QD. The diagonal coupling constants are

$$
\begin{aligned}
& F_{n n}(\boldsymbol{k})= \\
& {\left[\sigma_{\mathrm{e}}\left(1-\xi_{n}^{(e)} \frac{k_{\perp}^{2} l^{2}}{4}\right)-\sigma_{\mathrm{h}}\left(1-\xi_{n}^{(h)} \frac{k_{\perp}^{2} l^{2}}{4}\right)\right]} \\
& \times \sqrt{\frac{k}{2 \varrho v c_{l}}} \exp \left[-\frac{l_{z}^{2} k_{z}^{2}+l^{2} k_{\perp}^{2}}{4}\right],
\end{aligned}
$$

where $\xi_{n}^{(e / h)}=1$ if the electron/hole is in an excited state in the exciton state $n$ and $\xi_{n}^{(e / h)}=0$ otherwise. Here $v$ is the normalization volume, $k_{\perp / z}$ are momentum components in the $x y$ plane and along the $z$ axis, $\phi$ is the corresponding azimuthal angle, $\sigma_{\mathrm{e} / \mathrm{h}}$ are deformation potential constants for electrons/holes, $c_{l}$ is the speed of longitudinal sound, and $\varrho$ is the crystal density.

The phonon modes are described by the free phonon Hamiltonian

$$
H_{\mathrm{ph}}=\sum_{\boldsymbol{k}} \hbar \omega_{\boldsymbol{k}} b_{\boldsymbol{k}}^{\dagger} b_{\boldsymbol{k}}
$$

where $\omega_{\boldsymbol{k}}$ are the corresponding frequencies. We assume a linear dispersion relation for phonons.

In our numerical simulations, we take the parameters corresponding to large, flat self-assembled InAs/GaAs QDs: $\sigma_{\mathrm{e}}=7 \mathrm{eV}, \sigma_{\mathrm{h}}=-3.5 \mathrm{eV}, \rho=5350 \mathrm{~kg} / \mathrm{m}^{3}$, $c_{l}=5150 \mathrm{~m} / \mathrm{s}$, the wave function parameters $l=20 \mathrm{~nm}$, $l_{z}=1 \mathrm{~nm}$.

\section{SIMULATION METHOD AND RESULTS}

\section{A. Uncoupled QDs}

\section{Evolution equations}

The general Master equation in the Markov limit and in the interaction picture ${ }^{26}$ has the form

$$
\begin{aligned}
\dot{\rho}= & \pi \sum_{k l m n} e^{i\left(\omega_{k l}-\omega_{m n}\right) t} R_{l k m n}\left(\omega_{m n}\right) \\
& \times[|m\rangle\langle n|\rho| l\rangle\langle k|-| l\rangle\langle k \mid m\rangle\langle n| \rho]+\text { h.c. },
\end{aligned}
$$

where

$$
\begin{aligned}
R_{l k m n}(\omega) & =\frac{1}{\hbar^{2}} \sum_{\boldsymbol{k}} F_{l k}(\boldsymbol{k}) F_{n m}^{*}(\boldsymbol{k}) \\
& \times \delta\left(|\omega|-\omega_{\boldsymbol{k}}\right)\left|n_{B}(\omega)+1\right| .
\end{aligned}
$$

Note that $R_{l k n m}(\omega)=R_{m n k l}(\omega)=R_{n m l k}^{*}(\omega)$. This equation can be transformed to the Lindblad form (see Appendix A) and therefore preserves the physicality (in particular, positive definiteness) of the density matrix. Here $\omega_{m n}=\left(\epsilon_{n}-\epsilon_{m}\right) / \hbar$ and $n_{\mathrm{B}}(\omega)$ is the Bose distribution function. For the phonon-induced intraband transitions, the frequencies $\omega_{m n}$ are determined by the parameters $\Delta_{\mathrm{I} / \mathrm{II}}^{(e / h)}, \Delta_{\mathrm{g} / \mathrm{ex}}^{(e / h)}$ (see Fig. 11). In deriving Eq. (5), we
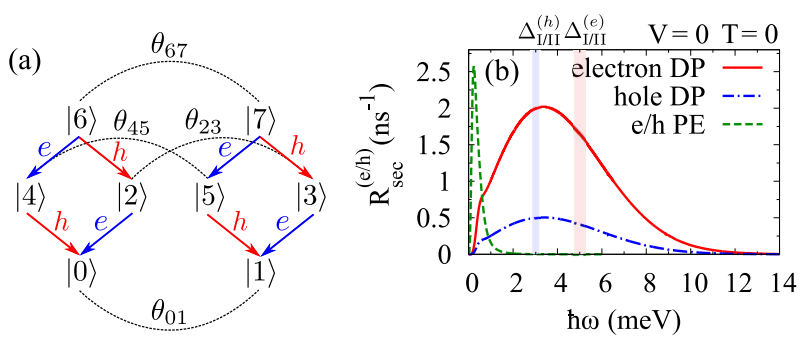

FIG. 2: (Color online) (a) The possible transitions between the states for the electron and the hole in the system. Red and blue arrows indicate transitions between exciton states involving electron and hole relaxation, respectively, while black dotted arrows show tunnel couplings between the states localized in different dots. (b) The secular phonon spectral densities at $T=0 \mathrm{~K}$, for an electron transition (red solid line), and hole transition (blue dashed line) respectively, coupled via deformation potential and for the electron/hole (green dashed line) coupled via piezoelectric field. The vertical stripes correspond to the range of changes of the energy-level differences in each $\operatorname{dot}\left(\Delta_{\mathrm{I} / \mathrm{II}}^{(e)}\right.$ and $\left.\Delta_{\mathrm{I} / \mathrm{II}}^{(h)}\right)$, for an electron and a hole, respectively.

have assumed that the reservoir memory is short compared to the time scales of the system evolution and restricted the description to times long compared to the memory time ${ }^{26}$. However, no secular approximation has been performed at this point.

In the case of no degeneracy between intraband transition energies $\left(\Delta_{\mathrm{I}}^{(e)}\right.$ and $\Delta_{\mathrm{I}}^{(h)}$ sufficiently different from $\Delta_{\mathrm{II}}^{(e)}$ and $\Delta_{\mathrm{II}}^{(h)}$, respectively), $\omega_{k l}-\omega_{m n}$ is large unless $k=m, l=n$, hence one can apply the secular approximation, neglecting terms with $k \neq m$ or $l \neq n$ and get a Lindblad equation in the form ${ }^{26}$

$$
\begin{aligned}
\dot{\rho} & = \\
& \pi \sum_{m n} R_{n m m n}\left(\omega_{m n}\right)[|m\rangle\langle n|\rho| n\rangle\langle m|-| l\rangle\langle k \mid m\rangle\langle n| \rho]+\text { h.c. } \\
& =2 \pi \sum_{m n} R_{n m m n}\left(\omega_{m n}\right)\left[|m\rangle\langle n|\rho| n\rangle\langle m|-\frac{1}{2}\{|n\rangle\langle n|, \rho\}\right](7)
\end{aligned}
$$

where $R_{n m m n}\left(\omega_{m n}\right)$ is the secular spectral density describing the phonon-assisted relaxation of either an electron (e.g. $\left.R_{2002}\left(\omega_{02}\right)\right)$ or a hole (e.g. $\left.R_{4004}\left(\omega_{04}\right)\right)$ within one dot, according to the electron or hole character of $F_{m n}(\boldsymbol{k})$. Thus, we only have two different secular spectral densities, denoted $R_{\mathrm{sec}}^{(e)}, R_{\mathrm{sec}}^{(h)}$. Both of them are presented in Fig. 2 with red solid and blue dash-dotted lines, respectively. They do not depend on the distance between the dots. For comparison, we plot in Fig. 2 also the spectral density for piezoelectric phonons, calculated using the standard model for piezoelectric carrier-phonon couplings 27 (green dashed line). As can be seen, this coupling contributes only at very low frequencies and can be neglected in view of much larger energy level spacings assumed in our model (vertical stripes in Fig. 2).

From Eq. (7) we get for the inter-dot coherence in the 

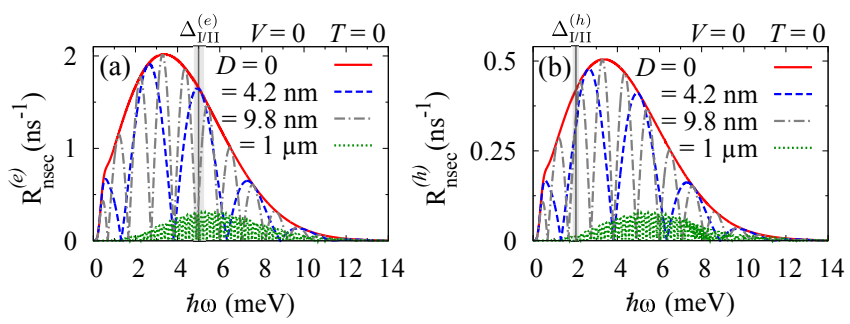

FIG. 3: (Color online) The non-secular phonon spectral densities at $T=0 \mathrm{~K}$ for uncoupled QDs, for an electron transition (a), and hole transition (b). Different lines correspond to the three values of the inter-dot distance, as shown. Here, the vertical gray lines correspond to the values of the energy-level differences used in our simulations in Sec. III A $\left(\Delta_{\mathrm{I} / \mathrm{II}}^{(e)}=5 \mathrm{meV}\right.$ and $\Delta_{\mathrm{I} / \mathrm{II}}^{(h)}=2 \mathrm{meV}$, for the electron and the hole, respectively).

ground state

$$
\frac{d}{d t}\langle 0|\rho| 1\rangle=-\frac{1}{2} \sum_{m}\left(\Gamma_{0 m}+\Gamma_{1 m}\right)\langle 0|\rho| 1\rangle,
$$

where $\Gamma_{n m}=2 \pi R_{n m m n}\left(\omega_{m n}\right)$. In this case we have a purely exponential decay and no coherence can appear dynamically.

Now, let us assume that $\omega_{k l}-\omega_{m n}$ can be small for pairs of transitions $(k l)$ and $(m n)$ corresponding to relaxation of a given particle (electron or hole) in different dots. Consistently with this, let us keep the corresponding terms in Eq. (5). For example, for $(m n)=(02)$ the terms with $(k l)=(02)$ and $(k l)=(13)$ are kept (both corresponding to electron relaxation in the two dots with the hole in the ground shell). According to Fig. 1] this condition corresponds to the degeneracy of intraband transition energies $\Delta_{\mathrm{I}}^{(e / h)} \approx \Delta_{\mathrm{II}}^{(e / h)}$. Now the equation of motion for the ground-state spatial coherence of interest is

$$
\begin{aligned}
& \frac{d}{d t}\langle 0|\rho| 1\rangle= \\
& \quad-\frac{1}{2} \sum_{m}\left(\Gamma_{0 m}+\Gamma_{1 m}\right)\langle 0|\rho| 1\rangle \\
& \quad+\pi \sum_{l n}\left[R_{l 10 n}\left(\omega_{0 n}\right)+R_{1 \ln 0}\left(\omega_{1 l}\right)\right] e^{i\left(\omega_{1 l}-\omega_{0 n}\right) t}\langle n|\rho| l\rangle
\end{aligned}
$$

where $(l, n)=(3,2)$ or $(5,4)$, corresponding to electron and hole relaxation, respectively. For the geometry chosen in our model, the non-secular spectral densities $R_{l 10 n}(\omega)$ are real and again we have only two different non-secular spectral densities, depending on whether the transitions $l \rightarrow 1$ and $n \rightarrow 0$ involve an electron or hole relaxation. They will be denoted by $R_{\text {nsec }}^{(e / h)}$. For this kind of a non-secular spectral density, the expression in Eq. (6) contains a factor $\cos \left(k_{z} D\right)$ (originating from the phase factor in Eq. (4)) depending on the distance be-
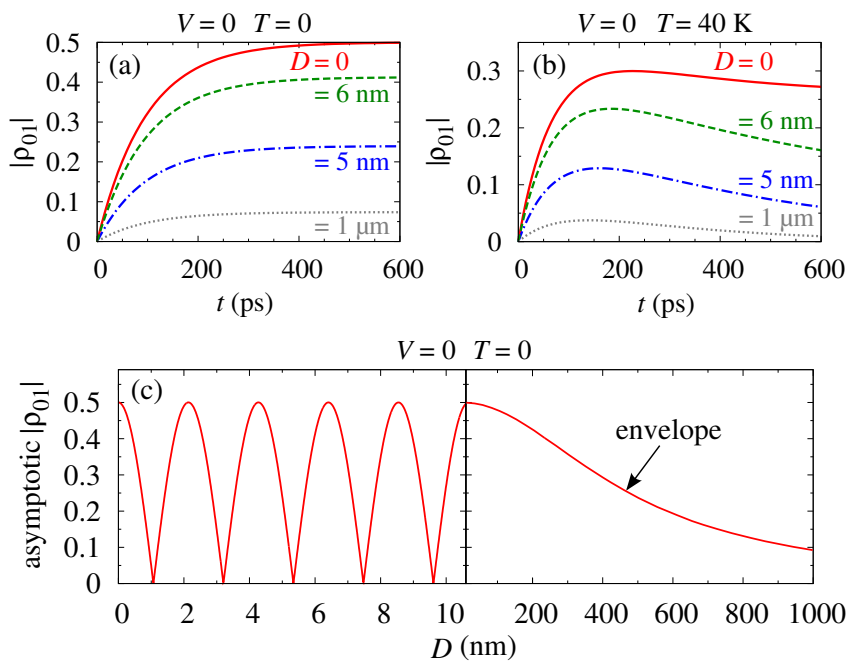

FIG. 4: (Color online) (a,b) The evolution of the spatial coherence for uncoupled QDs in the resonant case $\left(\Delta_{\mathrm{I} / \mathrm{II}}^{(e)}=\right.$ $5 \mathrm{meV}$ and $\Delta_{\mathrm{I} / \mathrm{II}}^{(h)}=2 \mathrm{meV}$ ) at different temperatures and at various inter-dot distances. (c) Left panel: The long-time asymptotic values of the spatial coherence for uncoupled QDs as a function of the inter-dot distance. Right panel: The envelope of the oscillations of the asymptotic value of $\rho_{01}$ at larger distances.

tween the dots,

$$
\begin{aligned}
\mathrm{R}_{\mathrm{nsec}}^{(e / h)}(\omega)= & \frac{1}{\hbar^{2}} \sum_{\boldsymbol{k}}\left|F_{e / h}(\boldsymbol{k})\right|^{2} \cos \left(k_{z} D\right) \\
& \times \delta\left(|\omega|-\omega_{\boldsymbol{k}}\right)\left|n_{B}(\omega)+1\right| .
\end{aligned}
$$

This oscillating factor in the integrand leads to oscillations in the spectral densities as shown in Fig. 3 .

The second term in Eq. (9) describes the transfer of coherence from $\rho_{23}$ (electron delocalized between the QDs in the excited shell) and $\rho_{45}$ (hole in the excited shell) to the ground state coherence $\rho_{01}$. Let us note that this term contains the non-secular spectral densities dependent on the inter-dot distance, as given in Eq. (10), hence the resulting transfer of the coherence is sensitive to the spatial separation of the dots.

\section{Results and discussion}

Let us first study the simplest situation, where the system is prepared in the state $(|2\rangle+|3\rangle) / \sqrt{2}$ (electron at the excited level) in the case of exact degeneracy $\left(\omega_{13}=\right.$ $\omega_{02}$, that is, the intraband excitation energies for the electron are the same in both QDs, $\left.\Delta_{\mathrm{I}}^{(e)}=\Delta_{\mathrm{II}}^{(e)}\right)$. In this case, the solution of Eq. (9) for the spatial coherence $\rho_{01}$ at $T=0 \mathrm{~K}$ reads

$$
\rho_{01}=c[1-\exp (-\gamma t)]
$$



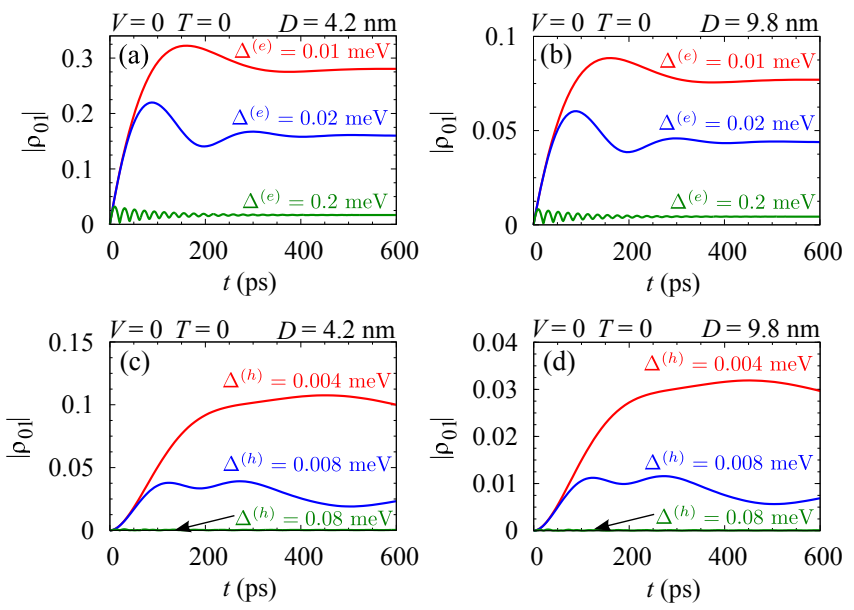

FIG. 5: (Color online) The evolution of the spatial coherence for uncoupled QDs at a constant inter-dot distance for a few values of the parameters characterizing the inhomogeneity of the DQD $\left(\operatorname{denoted} \Delta^{(e)}=\Delta_{\mathrm{II}}^{(e)}-\Delta_{\mathrm{I}}^{(e)}\right.$ and $\Delta^{(h)}=\Delta_{\mathrm{II}}^{(h)}-\Delta_{\mathrm{I}}^{(h)}$ ) for the initial state $(|2\rangle+|3\rangle) / \sqrt{2}$ (electron excited) (a,b) and for the initial state $(|6\rangle+|7\rangle) / \sqrt{2}$ (electron and hole excited) $(\mathrm{c}, \mathrm{d})$. The values of $\Delta^{(e)}$ in $(\mathrm{c}, \mathrm{d})$ are the same as for the corresponding lines in $(\mathrm{a}, \mathrm{b})$.

where $\gamma=\pi\left(R_{2002}\left(\omega_{02}\right)+R_{3113}\left(\omega_{13}\right)\right)=2 \pi R_{\sec }^{(e)}\left(\omega_{02}\right)$ and

$$
c=\frac{1}{2} \frac{R_{3102}\left(\omega_{02}\right)+R_{1320}\left(\omega_{13}\right)}{R_{2002}\left(\omega_{02}\right)+R_{3113}\left(\omega_{13}\right)}=\frac{1}{2} \frac{R_{\mathrm{nsec}}^{(e)}\left(\omega_{02}\right)}{R_{\mathrm{sec}}^{(e)}\left(\omega_{02}\right)} .
$$

The results are presented in Fig. 4 for a few values of the inter-dot distance. As follows from Eq. (11), the rate of the coherence transfer is directly related to the relaxation rate. However, the maximum value reached by the spatial coherence depends on the ratio of the magnitude of the additional term in Eq. (9) to the usual carrier relaxation (or thermalization) rates, which reflects the competition between the coherence transfer and its decay in the initial state due to relaxation. At $T=0 \mathrm{~K}$ the transitions from the ground state shell to the excited shells are fully suppressed and the ground state coherence reaches a stable asymptotic value at long times (Fig. 廿(a)). Due to the oscillating character of the non-secular spectral density $R_{\text {nsec }}$, only at certain inter-dot distances this asymptotic value reaches its maximum which, for flat and not very

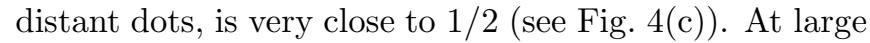
distances, the oscillating factor in Eq. (10) suppresses the result of the summation, reducing the coherence transfer rate and the maximum achievable value of $\rho_{01}$. At higher temperatures the ground state spatial coherence is further suppressed due to thermally activated processes, which eventually lead to its decay, as shown in Fig. 4(b), where we present the results of numerical simulations at $T=40 \mathrm{~K}$.

We have so far assumed the perfect resonance condition which is unlikely in a real system. The actual importance of the coherence-transfer term in Eq. (9) depends on the relation between the frequency differences
$\omega_{13}-\omega_{02}=\Delta_{\mathrm{II}}^{(e)}-\Delta_{\mathrm{I}}^{(e)}$ and $\omega_{15}-\omega_{04}=\Delta_{\mathrm{II}}^{(h)}-\Delta_{\mathrm{I}}^{(h)}($ see Fig. 1) on one side, and the typical rates of the system evolution in the interaction picture (coherence transfer and relaxation rates) on the other side. Therefore, we now proceed to the discussion of a more general case, in which the intraband energy splittings are not exactly equal. This is shown in Fig. 5(a), where we present the evolution of the ground state spatial coherence for a few values of the energy-level difference between the dots, for the initial state $(|2\rangle+|3\rangle) / \sqrt{2}$. We start from the resonant values $\left(\Delta_{\mathrm{I} / \mathrm{II}}^{(e)}=5 \mathrm{meV}\right.$ and $\left.\Delta_{\mathrm{I} / \mathrm{II}}^{(h)}=2 \mathrm{meV}\right)$, then we shift the energy levels away from resonance in a symmetric way. In Fig. 5 (a) we choose the distance $D=4.2 \mathrm{~nm}$, for which a local maximum of the non-secular spectral density for the electron is located at the chosen energy values (blue dashed line and vertical bar in Fig. 33(a)), while in Fig. 5 (b), $D=9.8 \mathrm{~nm}$ corresponds to a much lower value of the non-secular spectral density at the relevant energy (gray dash-dotted line in Fig. 3(a)) and, in consequence, to a lower asymptotic value of the ground state coherence. It is clear that even slightly different dots result in a weak spatial coherence. This is due to the oscillating term in Eq. (5) which supresses the transfer of spatial coherence as soon as the intraband transition energy difference, $\omega_{k l}-\omega_{m n}$, becomes comparable with the coherence transfer rates. This oscillating factor leads to oscillations in the ground state spatial coherence in place of the steady growth appearing at exact resonance. With an increasing inhomogeneity of the QDs, the oscillations get faster and finally the term responsible for the transfer of coherence becomes effectively averaged to zero.

Up to now, our discussion was limited to the direct coherence transfer by a single-step relaxation (from $\rho_{23}$ to $\left.\rho_{01}\right)$. In the same way, the coherence $\rho_{01}$ can be fed by $\rho_{45}$. The coherences $\rho_{23}$ and $\rho_{45}$ are driven by the coherence $\rho_{67}$ in a similar way. Let us now proceed to the general situation in which the initial state is formed by both the electron and the hole in the excited states, that is, the fully excited initial state $(|6\rangle+|7\rangle) / \sqrt{2}$ (which can be achieved by an optical excitation tuned to the p-shell of confined states). The evolution of the ground

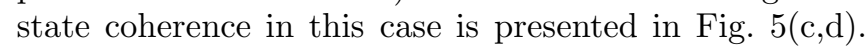
Now, the evolution of the spatial coherence saturates on a longer time scale. This is due to the phonon relaxation of the hole, which is typically slower than the relaxation of the electron. In addition, large part of the spatial coherence is lost during hole relaxation, hence the attained asymptotic values are lower.

\section{B. Coupled QDs}

\section{System states}

When the interaction between the dots is included, the system evolution is most conveniently described in the basis of eigenstates of $H_{\mathrm{DQD}}$. These single-exciton 
eigenstates result from the tunnel coupling between the exciton states in different dots and can be written as

$$
\begin{aligned}
& |\widetilde{m}\rangle=\cos \frac{\theta_{m n}}{2}|m\rangle+\sin \frac{\theta_{m n}}{2}|n\rangle, \\
& |\widetilde{n}\rangle=-\sin \frac{\theta_{m n}}{2}|m\rangle+\cos \frac{\theta_{m n}}{2}|n\rangle .
\end{aligned}
$$

Here $m, n$ describe the coupled even and odd states, respectively (that is, a pair of states with a carrier in different dots but in the same energy shell), $\theta_{m n}$ is the mixing angle, defined by $\tan \theta_{m n}=2 V_{m} /\left(\epsilon_{m}-\epsilon_{n}\right)$, where $\epsilon_{m / n}$ denotes the energy levels in the two dots without the coupling. The energies of the states $|\widetilde{m}\rangle$ and $|\widetilde{n}\rangle$ are denoted by $E_{m}$ and $E_{n}$, respectively, where

$$
E_{n / m}=\frac{\epsilon_{m}+\epsilon_{n}}{2} \pm \frac{1}{2} \Delta E_{n m}
$$

and

$$
\Delta E_{n m}=\sqrt{\left(\epsilon_{n}-\epsilon_{m}\right)^{2}+4 V^{2}} .
$$

The spatial coherence in the ground state is related to the density matrix elements in the eigenstate basis by

$$
\begin{aligned}
\langle 0|\rho| 1\rangle= & \frac{1}{2} \sin \theta_{01}(\langle\widetilde{0}|\rho| \widetilde{0}\rangle-\langle\widetilde{1}|\rho| \widetilde{1}\rangle) \\
& +\cos ^{2} \frac{\theta_{01}}{2}\langle\widetilde{0}|\rho| \widetilde{1}\rangle-\sin ^{2} \frac{\theta_{01}}{2}\langle\widetilde{1}|\rho| \widetilde{0}\rangle .
\end{aligned}
$$

It is clear that the spatial coherence can appear in the ground state as a result of phonon-induced relaxation even in the absence of the special degeneracy discussed above, which is a trivial consequence of the fact that the ground state of coupled dots is coherently delocalized by itself. At $T=0 \mathrm{~K}$, when the system relaxes to the ground state, this coherence is $(1 / 2) \sin \theta_{01}$. At finite temperatures, the equilibrium state of the system is

$$
\rho=\sum_{n} p_{n}|\tilde{n}\rangle\langle\tilde{n}|,
$$

where $p_{n}=Z^{-1} \exp \left(-E_{n} / k_{B} T\right)$ and $Z=$ $\sum_{n} \exp \left[-E_{n} / k_{B} T\right]$. According to Eq. (15) this yields the degree of coherence

$$
\begin{aligned}
\langle 0|\rho| 1\rangle & =\frac{1}{2} \sin \theta_{01}\left(p_{0}-p_{1}\right) \\
& =\frac{1}{2} \sin \theta_{01}\left(p_{0}+p_{1}\right) \tanh \frac{E_{1}-E_{0}}{2 k_{B} T} .
\end{aligned}
$$

Hence, the coherence at equilibrium depends on the mixing angle (reflecting the relative coupling strength), the total occupation probability of the ground shell, and the thermal redistribution of occupations between the two states in the ground shell. In a certain range of temperatures, when the occupations of the higher shells can be neglected (hence $p_{0}+p_{1} \approx 1$ ), the ground state spatial
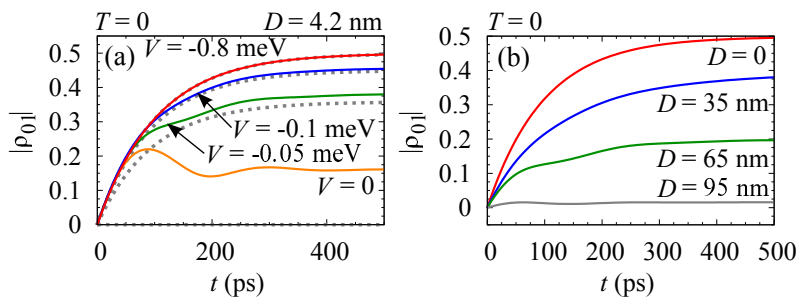

FIG. 6: (Color online) The evolution of the spatial coherence for coupled QDs, with $\Delta^{(e)}=0.02 \mathrm{meV}$ and $\Delta_{\mathrm{g}}^{(e)}=$ $-0.05 \mathrm{meV}$, for the initial state $(|2\rangle+|3\rangle) / \sqrt{2}$ (electron excited). (a) For selected values of the interaction $V$ and a constant inter-dot distance. The gray dotted lines represent the results obtained from the Lindblad equation (secular approximation) for the same values of the parameters. (b) For the interaction $V$ that depends on the distance between the dots.

coherence is determined by the last factor in the above equation.

For the numerical simulations of the evolution of the density matrix for coupled QDs, we use the Master equation as in Eq. (9), but now in the eigenstate basis and with the corresponding spectral densities $\widetilde{R}$ (related to those in the original basis as described in detail in the Appendix B). The energy splittings $\Delta_{\mathrm{I} / \mathrm{II}}^{(e)}$ and $\Delta_{\mathrm{I} / \mathrm{II}}^{(h)}$ (see Fig. (1) are obtained by the symmetric displacement of the states with respect to $\Delta_{\mathrm{I}}^{(e)}=\Delta_{\mathrm{II}}^{(e)}=5 \mathrm{meV}$ for the electron and $\Delta_{\mathrm{I}}^{(h)}=\Delta_{\mathrm{II}}^{(h)}=2 \mathrm{meV}$ for the hole, while the energy difference between the ground states in the dots is constant and has the value $\Delta_{\mathrm{g}}^{(e)}=-0.05 \mathrm{meV}$ and $\Delta_{\mathrm{g}}^{(h)}=-0.008 \mathrm{meV}$ for the electron and the hole, respectively.

\section{Simulation results and discussion}

Again, for the sake of clarity and simplicity, we start our discussion from the case of the initial state $(|2\rangle+$ $|3\rangle) / \sqrt{2}$ (only electron excited). The results of these simulations are shown in Fig. 6. To simplify the discussion, in Fig. 6(a) we fixed the inter-dot distance and only increase the coupling. For the chosen values of the parameters describing the inhomogeneity of the QDs and for a strong coupling $\left(V_{m}=V=-0.8 \mathrm{meV}\right)$, the simulation results obtained from the Lindblad equation, that is, in the secular approximation (gray dotted line), are in exact accordance with the simulation results obtained from the more general Master equation (red solid line). This means that the evolution of spatial coherence in this regime is dominated by simple relaxation to the coherently delocalized ground state. For lower values of the coupling the system reaches the equilibrium value of the coherence, with a correction to the Lindblad dynamics that grows with decreasing coupling and with some weak oscillations. These corrections are due to the growing 
role of the phonon-induced transfer of coherence from the upper levels. These oscillations do not appear in simulation results obtained in the secular approximation for the same values of the parameters (gray dotted lines in Fig. 6(a)) even though the main trend of the evolution of coherence as well as the asymptotic value are reproduced correctly unless the coupling becomes very weak.

In Fig. 6(b) we present the evolution of the spatial coherence in coupled QDs at $T=0 \mathrm{~K}$, when the coupling is related to the distance between the dots. For this dependence we choose the simplest exponential model ${ }^{28}, V=$ $-V_{0} \exp \left(-D / D_{0}\right)$, with the amplitude $V_{0}=0.5 \mathrm{meV}$ and the range $D_{0}=20 \mathrm{~nm}$. For distant dots (about hundreds of nanometers and more), the coupling vanishes, hence the equilibrium value of the ground state coherence decreases and the system reaches the limit of uncoupled dots described in Sec. III A, For closely placed dots, one essentially deals with a relaxation process to the ground state, hence the ground-state spatial coherence reaches higher values. Weak oscillations visible for intermediate distances are a fingerprint of the coherence transfer contribution to the process, which overall remains dominated by relaxation between the eigenstates. Obviously, the everlasting coherence in our simulation results is due to the lack of exciton decay in our model and, in a real system, will be limited at longer times by radiative recombination (see Appendix $\mathrm{C}$ for a brief discussion of these effects).

In Fig. 7(a) we show the impact of the relaxation within the ground shell on the system dynamics. Again, we choose the initial state with the excited electron only, and we fix the inter-dot distance. For better clarity, we keep also constant values of the intra-dot energy-level differences $\left(\Delta_{\mathrm{I}}^{(e)}=3.45 \mathrm{meV}, \Delta_{\mathrm{II}}^{(e)}=3.55 \mathrm{meV}\right)$. We consider five scenarios with different relaxation rates of the electron within and between the dots. The spectral densities relevant for the discussion of the results in Fig. 7(a) are presented in Fig. 7(b,c,d) and the associated occupation dynamics for the five cases to be analyzed is plotted quantitatively and represented diagrammatically in Fig. 8 .

We start our discussion from strongly coupled QDs with the mixing angle $\theta_{m n}$ close to $\pi / 2$. The spectral densities $\widetilde{R}_{1001}$ and $\widetilde{R}_{3003}$ (see the Appendix B) involved in the rate of the $\widetilde{n} \rightarrow \widetilde{m}$ transition at $T=0 \mathrm{~K}$ $\left(\widetilde{\Gamma}_{n m}=2 \pi \widetilde{R}_{n m m n}\left(\Delta E_{n m} / \hbar\right)\right)$ for this case are presented in Fig. Z(b). The initial state in this case is nearly identical with the eigenstate $|\widetilde{2}\rangle$, which is the lower-energy state of the excited electron shell. The evolution of the ground state spatial coherence in this case will depend on the relative values of the transition rates between this state and the two states in the ground shell of the DQD. Many scenarios are possible, out of which we discuss the two most characteristic ones. The red line in Fig. 7(a) $\left(\Delta_{g}^{(e)}=-0.05 \mathrm{meV}, V=-0.8 \mathrm{meV}, \theta_{01}=88.21^{\circ}\right.$, $\left.\theta_{23}=84.64^{\circ}\right)$ represents the evolution of the groundstate spatial coherence with fast relaxation between the
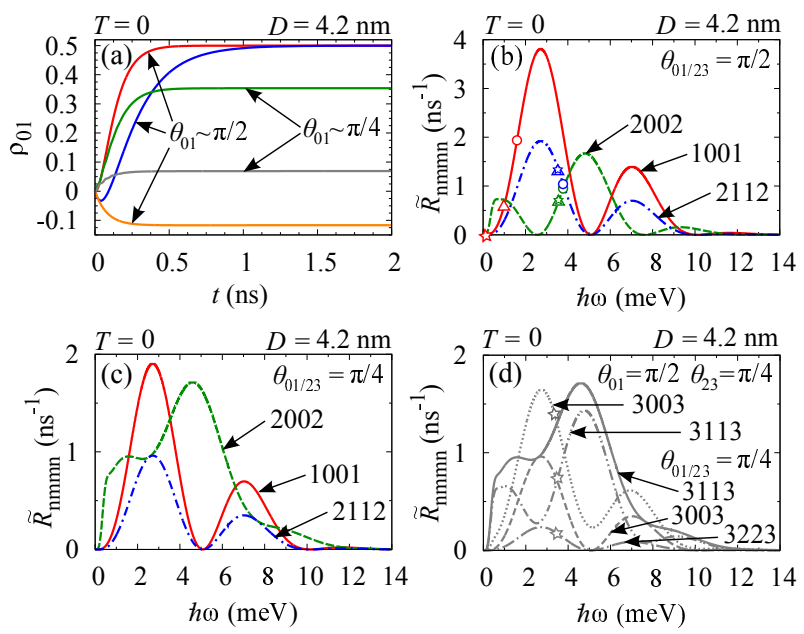

FIG. 7: (Color online) (a) The evolution of the spatial coherence for coupled QDs at a constant inter-dot distance. (b,c,d) The phonon spectral densities describing the electron relaxation within the dots, and the electron transfer between the states in the dots. The marks in (b,d) (colored circle, triangle and star) denote the transition energies corresponding to the cases with the red, blue and orange lines in panel (a), respectively.

two states in the ground shell (case (a) in Fig. 8). In this case, the occupation of the ground states changes fast and the spatial coherence reaches its asymptotic value in a short time. During the evolution one can observe only a small transient occupation of the state $|\widetilde{1}\rangle$ (see Fig. $8(\mathrm{a}$ (a)).

The blue line in Fig. [7(a) $\left(\Delta_{g}^{(e)}=-0.03 \mathrm{meV}, V=\right.$ $\left.-0.5 \mathrm{meV}, \theta_{01}=88.17^{\circ}, \theta_{23}=82.48^{\circ}\right)$ corresponds to the situation where the efficiency of the carrier transfer between the ground states is lower as compared to the relaxation to the state $|\widetilde{1}\rangle$ (see Fig. $[8(b)$ ). Although the system still finally relaxes to the delocalized ground state $|\overline{0}\rangle$ with the maximum value of the spatial coherence of $1 / 2$, the final ground-state spatial coherence saturates after a much longer time.

Now, we focus on weakly coupled QDs, where initially both of the excited-shell eigenstates are occupied. In such a situation, the efficiency of the carrier transfer between the dots strongly affects the value of the groundstate spatial coherence. In the limiting case of vanishing relaxation within the ground shell, the system state at $T=0 \mathrm{~K}$ is asymptotically frozen in a mixture of the state $|\widetilde{0}\rangle$ and $|\widetilde{1}\rangle$, with relative probabilities determined by the relaxation rates from $|\widetilde{3}\rangle$ and $|\widetilde{2}\rangle$ to these two eigenstates. Since the "anti-bonding" state $|\widetilde{1}\rangle$ contributes negative spatial coherence, the resulting value of this coherence may in principle achieve any value between $-1 / 2$ and $1 / 2$. An example is shown by the orange line in Fig. 7 (a) $\left(\Delta_{g}^{(e)}=-0.001 \mathrm{meV}, V=-0.05 \mathrm{meV}\right.$, $\theta_{01}=89.43^{\circ}, \theta_{23}=44.71^{\circ}$ ) and in Fig. 8(c). At sufficiently high temperatures, this state would become metastable and decay to the ground state $|\widetilde{0}\rangle$ due to thermally 

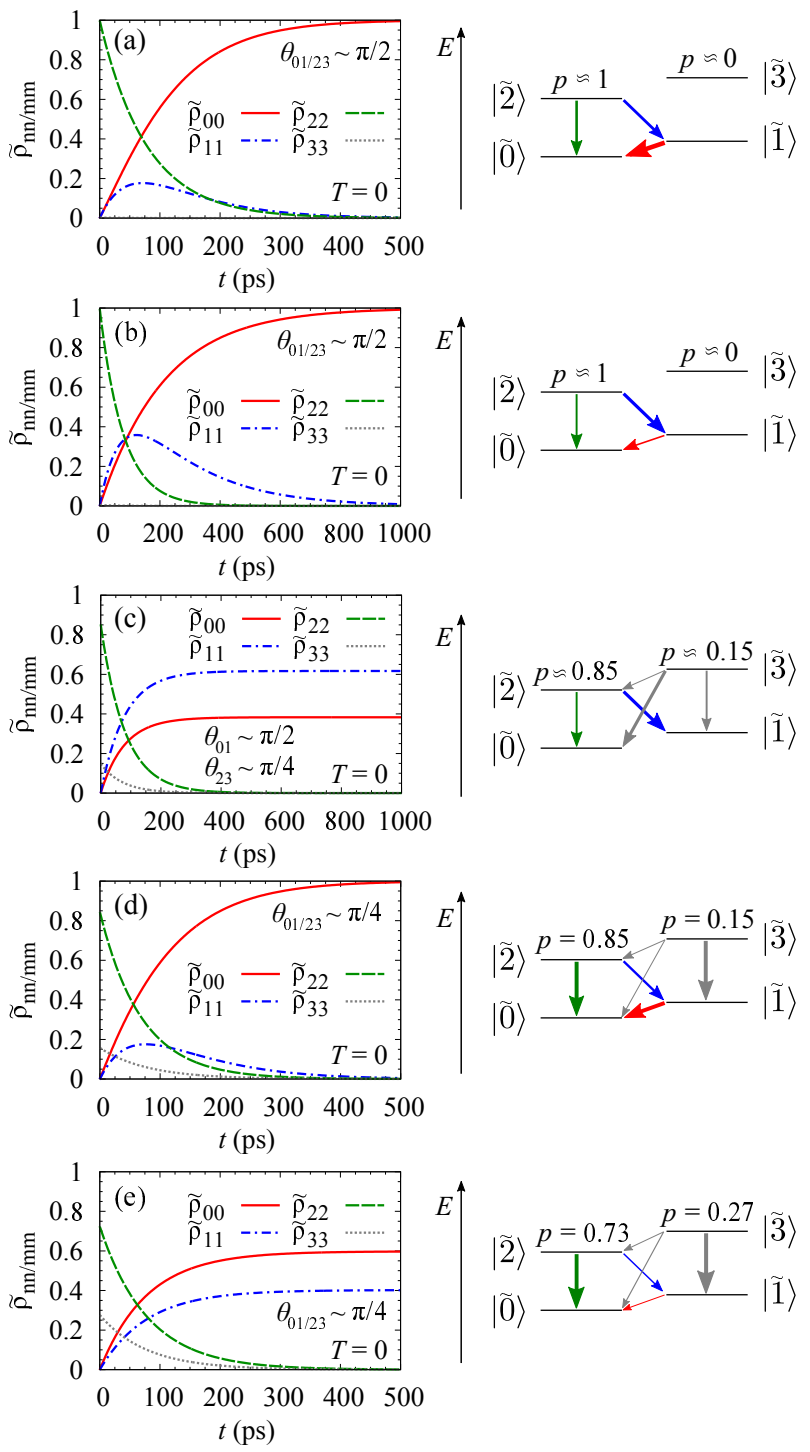

FIG. 8: (Color online) The occupation of the eigenstates at $T=0 \mathrm{~K}$ (left panel) and the diagram of the transition rates between the states (right panel) for the dynamical scenarios studied in the text. The color of arrows corresponds to the spectral densities presented in Fig. 7(b,c,d), while their thickness denotes the efficiency of the relaxation process, and $p$ is the initial probability of the occupation of the excited states.

activated transitions via the excited states.

The evolution in the case of weakly coupled QDs is shown in Fig. Z7(a) by the green line (fast carrier transfer between the dots, $\Delta_{g}^{(e)}=-1.6 \mathrm{meV}, V=-0.8 \mathrm{meV}$, $\theta_{01}=45^{\circ}, \theta_{23}=43.26^{\circ}$ ) and gray line (slow transfer, $\Delta_{g}^{(e)}=-0.1 \mathrm{meV}, V=-0.05 \mathrm{meV}, \theta_{01}=45^{\circ}$, $\left.\theta_{23}=26.57^{\circ}\right)$. The spectral densities relevant to these cases are shown in Fig. 7( $(\mathrm{c}, \mathrm{d})$. In the case of fast transfer, the electron transfer between the states in the ground shell is relatively efficient (see Fig. 8(d)) and the final ground-state spatial coherence quickly reaches its asymptotic value as in Eq. (17). In the other case, when the
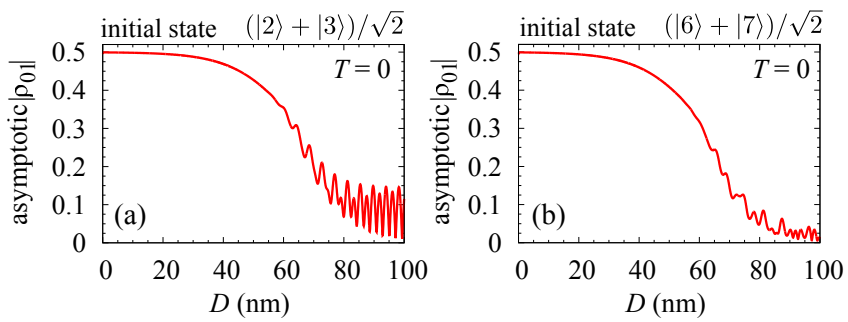

FIG. 9: (Color online) The asymptotic values of the spatial coherence between the two lowest exciton states in the system of coupled and non-identical QDs at $T=0 \mathrm{~K}$. (a) For the initial state $(|2\rangle+|3\rangle) / \sqrt{2}$ (electron excited), $\Delta^{(e)}=0.02 \mathrm{meV}$. (b) For the initial state $(|6\rangle+|7\rangle) / \sqrt{2}$ (both electron and hole excited), $\Delta^{(e)}=0.02 \mathrm{meV}, \Delta^{(h)}=0.008 \mathrm{meV}$.

transfer within the ground shell is extremely slow (see Fig. 8(e)), the final degree of coherence results from the coherence transfer process, with the final value yielded by the interplay of the occupation relaxation and coherence transfer rates, as discussed in the previous section. In the particular case shown in Fig. 17(a) (gray line), this value is lower than that associated with the ground state (green line).

In Fig. 9 we present the long-time asymptotic values of the spatial coherence in coupled QDs at $T=0 \mathrm{~K}$, as a function of the inter-dot distance. For small distances, the effect is dominated by the ground state coherence. Since the mixing angle decreases with vanishing coupling, so does the amount of spatial coherence in the ground state and, as a result, the final degree of spatial coherence decreases with the inter-dot distance. For larger distances between the dots the efficiency of the coherence transfer is most important (uncoupled dots limit, see Sec. (IIIA), hence the asymptotic value oscillates with the distance, in view of the corresponding form of the spectral density (as in Fig. 4(c), but with a smaller amplitude because of non-identical dots). Furthermore, the coupling becomes smaller than the energy mismatch and the resulting maximum value of the coherence is reduced. The resulting dependence on the distance is similar for the initially excited electron (Fig. 9(a)) and for both the electron and the hole excited (Fig. 9(b)).

An important feature of phonon-related effects is their strong temperature dependence. In Fig. 10 we show the evolution of the spatial coherence in coupled QDs at a few values of the temperature. In Fig. 10(a) we present the results for the initial state $(|2\rangle+|3\rangle) / \sqrt{2}$ (excited electron). In Fig. 10(b), results for the initial state $(|6\rangle+|7\rangle) / \sqrt{2}$ are presented where, at the beginning, the electron and hole are in their excited states. The thermal distribution of the occupations reduces the asymptotic degree of coherence in accordance with Eq. (17). At a low temperature (blue dashed line), mostly the ground states are occupied. At higher temperatures the occupations are distributed between the ground and excited states, hence the ground state coherence is suppressed. 

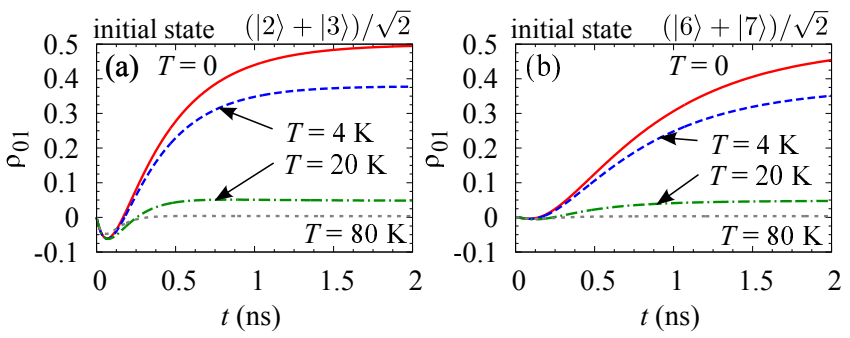

FIG. 10: (Color online) The evolution of the spatial coherence between the two lowest exciton states in the system of nonidentical QDs at various temperatures. (a) $\Delta^{(e)}=0.02 \mathrm{meV}$. (b) $\Delta^{(e)}=0.02 \mathrm{meV}, \Delta^{(h)}=0.008 \mathrm{meV}$.

Let us note that in comparison with the electron, the hole has a lower value of the deformation potential constant. Hence, the phonon relaxation of the hole is slower and the resulting evolution of the spatial coherence in the case of both carriers initially excited saturates after a longer time.

\section{DISCUSSION AND CONCLUSIONS}

We have studied theoretically the evolution of the spatial coherence during relaxation between delocalized exciton states in the system consisting of two vertically stacked semiconductor QDs coupled to the acoustic phonons. We have shown that the coupling to phonons can lead to coherence transfer from a delocalized excited state to the ground state manifold of the DQD, which accompanies phonon-induced relaxation in this system. As we have found, the coherence transfer in a system of decoupled dots requires that the intraband energy spacings in the two dots should be close to each other. In the presence of coupling between the dots, some spatial coherence is always present in the system ground state. Hence, in the evolution, one deals with an interplay between the coherence transfer process (which is related to intra-dot relaxation), and the relaxation to the ground state (which involves inter-dot transfer, enabled by the coupling).

As a final element of our discussion, let us see to what extent the required degeneracy of intraband transition energies is likely to appear in a real system. The inhomogeneous broadening of ground state photoluminescence lines in QD ensembles is typically on the order of $10 \mathrm{meV}$. Apart from being composed of electron and hole contributions, this broadening is mostly due to the fluctuation in the QD size $H$ along the strongest confinement axis (i.e., the growth direction). Since the energy is proportional to inverse square of the size, the variation of the intraband transition energies, which depend on the lateral size $L$, is reduced by a factor $(H / L)^{2}$, that is, by one or two orders of magnitude. This estimate yields a typical inhomogeneity of electron intraband transition energies of $0.1 \mathrm{meV}$ and a correspondingly smaller value for the hole. These energies may lie even closer to each other in particular QD pairs. On the other hand, the critical value of the inhomogeneity that destroys the coherence transfer effect is related to the onset of validity of the secular approximation and hence depends on the typical relaxation rate. In smaller QDs, where the LO-phonon mediated relaxation is faster by an order of magnitude than in our present model, the tolerance of this effect against inhomogeneity will also be enhanced by an order of magnitude.

The present study was based on a minimal model with the aim to demonstrate that the process in question is possible in principle and to characterize the most general conditions that must be met for this process to appear. Obviously, it must be supplied with much more details concerning the actual system spectrum and the full set of phonon modes and processes that contribute to relaxation in a typical structure (most likely optical phonons and anharmonicity-related processes) in order to yield quantitative conclusions on the importance of the coherence transfer process under particular experimental conditions. In the detailed model, one might also include the exact structure of wave functions. In particular, the product form of the exciton wave functions is not strictly consistent with the assumption that the Coulomb attraction is larger than the differences between single particle levels. Including Coulomb-correlated exciton states would yield more accurate values of transition rates, although the general structure of the model and, therefore, the essential conclusions of our analysis would remain unaffected. Apart from the possible quantitative improvements of the model, already at the present stage of a "proof of principle", our results indicate that the interplay of the coupling and energy degeneracy is crucial not only for the appearance of collective emission itself $f^{3,5}$ but also for the coherence transfer during relaxation that is needed for the appearance of the coherent Dicke-like state in the ground shell of the DQD.

\section{Acknowledgments}

This work was supported by the grant No. 2011/01/B/ST3/02415 from the Polish National Science Centre.

\section{APPENDIX A: NON-SECULAR MASTER EQUATION PRESERVES PHYSICALITY}

In this Appendix we show that retaining the nonsecular terms in Eq. (5) does not break the physicality of the model in the sense that the density matrix remains positive-definite during the evolution generated by this master equation. We do this by explicitly transforming Eq. (6) into the Lindblad form, which is known to posses the required properties 26 .

For the sake of clarity, let us define $\sigma_{m n}=|m\rangle\langle n|$. We 
start by rewriting Eq. (6) in terms of the density matrix in the Schrödinger picture, $\rho_{\mathrm{S}}=e^{-i H_{0} t / \hbar} \rho e^{i H_{0} t / \hbar}$, where $H_{0}=\sum_{n} \epsilon_{n}|n\rangle\langle n|$. One finds

$$
\dot{\rho}_{\mathrm{S}}=-\frac{i}{\hbar}\left[H_{0}, \rho_{\mathrm{S}}\right]+\mathcal{L}_{\mathrm{S}}\left[\rho_{\mathrm{S}}\right]
$$

where

$$
\begin{aligned}
& \mathcal{L}_{\mathrm{S}}\left[\rho_{\mathrm{S}}\right]= \\
& \quad \pi \sum_{l k m n} R_{l k m n}\left(\omega_{m n}\right)\left(\sigma_{m n} \rho_{\mathrm{S}} \sigma_{k l}^{\dagger}-\sigma_{k l}^{\dagger} \sigma_{m n} \rho_{\mathrm{S}}\right)+\text { h.c. }
\end{aligned}
$$

By appropriately renaming the summation indices and using the symmetry relations for the spectral densities listed below Eq. (6), the dissipator can be written in the form

$$
\mathcal{L}_{\mathrm{S}}\left[\rho_{\mathrm{S}}\right]=-\frac{i}{\hbar}\left[H_{1}, \rho_{\mathrm{S}}\right]+\mathcal{L}^{\prime}\left[\rho_{\mathrm{S}}\right]
$$

where

$$
\mathcal{L}^{\prime}\left[\rho_{\mathrm{S}}\right]=2 \pi \sum_{k l m n} \Gamma_{(k l)(m n)}\left(\sigma_{m n} \rho_{\mathrm{S}} \sigma_{k l}^{\dagger}-\left\{\sigma_{k l}^{\dagger} \sigma_{m n}, \rho_{\mathrm{S}}\right\}\right)
$$

with $\Gamma_{(k l)(m n)}=\left[R_{l k m n}\left(\omega_{m n}\right)+R_{l k m n}\left(\omega_{k l}\right)\right] / 2$, and

$$
H_{1}=\frac{i}{2} \sum_{k l m n}\left[R_{l k m n}\left(\omega_{m n}\right)-R_{l k m n}\left(\omega_{k l}\right)\right] \sigma_{k l}^{\dagger} \sigma_{m n} .
$$

In view of the symmetries of the spectral densities, $H_{1}$ is a hermitian operator and the matrix of transition rates $\Gamma$ is also hermitian, that is, $\Gamma_{(k l)(m n)}=\Gamma_{(m n)(k l)}^{*}$. The dissipator $\mathcal{L}^{\prime}$ can therefore be brought to the standard Lindblad form ${ }^{26}$. One diagonalizes $\Gamma$ by a unitary matrix $U$,

$$
\sum_{k l m n} U_{(k l), \alpha}^{*} \Gamma_{(k l)(m n)} U_{(m n), \beta}=\gamma_{\alpha} \delta_{\alpha \beta}
$$

with $\sum_{k l} U_{(k l), \alpha} U_{(k l), \beta}^{*}=\delta_{\alpha \beta}$ and $\sum_{\alpha} U_{(k l), \alpha} U_{\left(k^{\prime} l^{\prime}\right), \alpha}^{*}=$ $\delta_{k k^{\prime}} \delta_{l l^{\prime}}$. Define $\sigma_{\alpha}=\sum_{k l} U_{(k l), \alpha}^{*} \sigma_{k l}$, that is, $\sigma_{k l}=$ $\sum_{\alpha} U_{(k l) \alpha} \sigma_{\alpha}$. In terms of these new operators one finds

$$
\mathcal{L}^{\prime}\left[\rho_{\mathrm{S}}\right]=\sum_{\alpha} \widetilde{\Gamma}_{\alpha}\left[\sigma_{\alpha} \rho_{\mathrm{S}} \sigma_{\alpha}^{\dagger}-\frac{1}{2}\left\{\sigma_{\alpha}^{\dagger} \sigma_{\alpha}, \rho_{\mathrm{S}}\right\}\right] .
$$

Hence, Eq. (6) in the Schrödinger picture has the form of the Lindblad equation

$$
\dot{\rho}_{\mathrm{S}}=-\frac{i}{\hbar}\left[H_{0}+H_{1}, \rho_{\mathrm{S}}\right]+\mathcal{L}^{\prime}\left[\rho_{\mathrm{S}}\right]
$$

and therefore preserves the physicality of the density matrix.

\section{APPENDIX B: SPECTRAL DENSITIES FOR COUPLED QDS}

In this appendix, we summarize the calculation of the spectral densities for a coupled system, which are related to those in the original basis, defined in Sec. IIIA. We focus on the secular and non-secular spectral densities for the electron relaxation. The same calculations can be repeated for the hole relaxation.

Upon transformation to the eigenbasis defined by Eqs. (12) and (13), Eq. (3) becomes

$$
\begin{aligned}
H_{\mathrm{c}-\mathrm{ph}} & =\sum_{(m n)}|\widetilde{m}\rangle \widetilde{n} \mid \sum_{\boldsymbol{k}} \widetilde{F}_{m n}(\boldsymbol{k})\left(b_{\boldsymbol{k}}+b_{-\boldsymbol{k}}^{\dagger}\right) \\
& +\sum_{\left(n^{\prime} n\right)}\left|\widetilde{n}^{\prime}\right\rangle\langle\widetilde{n}| \sum_{\boldsymbol{k}} \widetilde{F}_{n^{\prime} n}(\boldsymbol{k})\left(b_{\boldsymbol{k}}+b_{-\boldsymbol{k}}^{\dagger}\right) .
\end{aligned}
$$

The first term in the Hamiltonian is derived from the first term in Eq. (3) and corresponds to inter-shell transitions. The coupling between the QDs and the resulting state mixing opens new (cross-QD) relaxation channels, compared to Eq. (3), where only relaxation within a QD was possible. This is accounted for by a larger set of coupling constants, e.g.,

$$
\widetilde{F}_{02}(\boldsymbol{k})=\cos \frac{\theta_{01}}{2} \cos \frac{\theta_{23}}{2} F_{02}(\boldsymbol{k})+\sin \frac{\theta_{01}}{2} \sin \frac{\theta_{23}}{2} F_{13}(\boldsymbol{k}) .
$$

The second term, in which $\left[n^{\prime} n\right]$ denotes summation over pairs of states belonging to one shell, originates from the diagonal couplings which did not contribute to the dynamics in the uncoupled case in the Markov limit. In the presence of the coupling between the QDs, these diagonal terms yield transitions between the delocalized states that form a doublet within a given shell. For instance,

$$
\widetilde{F}_{01}(\boldsymbol{k})=\frac{1}{2} \sin \theta_{01}\left(F_{11}(\boldsymbol{k})-F_{00}(\boldsymbol{k})\right) .
$$

The spectral densities for the coupled case are then calculated according to Eq. (6) using the transformed coupling constants. As a result, one obtains, e.g., for the spectral densities most relevant to the discussion in Sec. IIIB,

$$
\begin{gathered}
\widetilde{\mathrm{R}}_{2002}(\omega)=\widetilde{\mathrm{R}}_{3113}(\omega)=C_{1} \mathrm{R}_{\mathrm{sec}}^{(e)}(\omega)+C_{2} \mathrm{R}_{\mathrm{nsec}}^{(e)}(\omega), \\
\widetilde{\mathrm{R}}_{2112}(\omega)=\widetilde{\mathrm{R}}_{3003}(\omega)=C_{3} \mathrm{R}_{\mathrm{sec}}^{(e)}(\omega)-C_{2} \mathrm{R}_{\mathrm{nsec}}^{(e)}(\omega), \\
\widetilde{\mathrm{R}}_{1001}(\omega)=\frac{1}{4} \sin ^{2} \theta_{01}\left[\mathrm{R}_{0000}^{(e)}(\omega)+\mathrm{R}_{1111}^{(e)}(\omega)\right. \\
\left.-\mathrm{R}_{1100}^{(e)}(\omega)-\mathrm{R}_{0011}^{(e)}(\omega)\right],
\end{gathered}
$$

where

$$
\begin{aligned}
& C_{1}=\sin ^{2} \frac{\theta_{01}}{2} \sin ^{2} \frac{\theta_{23}}{2}+\cos ^{2} \frac{\theta_{01}}{2} \cos ^{2} \frac{\theta_{23}}{2}, \\
& C_{2}=\frac{1}{2} \sin \theta_{01} \sin \theta_{23}, \\
& C_{3}=\sin ^{2} \frac{\theta_{01}}{2} \cos ^{2} \frac{\theta_{23}}{2}+\cos ^{2} \frac{\theta_{01}}{2} \sin ^{2} \frac{\theta_{23}}{2} .
\end{aligned}
$$



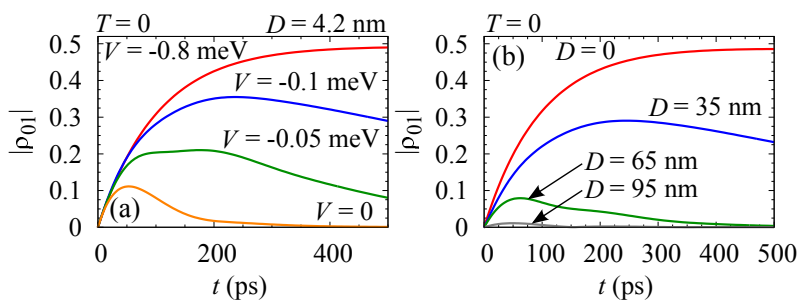

FIG. 11: (Color online) The evolution of the spatial coherence for coupled QDs (including the radiative recombination of exciton), with the same values of the parameters $\Delta^{(e)}$ and $\Delta_{\mathrm{g}}^{(e)}$ as in Fig. 6] again for the initial state with the excited electron only $((|2\rangle+|3\rangle) / \sqrt{2})$. (a) For selected values of the interaction $V$ and a constant inter-dot distance. (b) For the interaction $V$ that depends on the distance between the dots.

\section{APPENDIX C: THE EFFECT OF FINITE EXCITON LIFE TIME}

Spontaneous emission (radiative recombination of excitons) is modeled by the Lindblad equation, written in the basis of exciton eigenstates $|\widetilde{l}\rangle$ [Eqs. (12) and (13)],

$$
\begin{aligned}
& \mathcal{L}_{\mathrm{rad}}[\rho]= \\
& \quad \sum_{l} \gamma_{l}\left[\Sigma_{l} \rho \Sigma_{l}^{\dagger}-\frac{1}{2}\left(\Sigma_{l}^{\dagger} \Sigma_{l} \rho+\rho \Sigma_{l}^{\dagger} \Sigma_{l}\right)\right]
\end{aligned}
$$

where $\Sigma_{l}$ annihilates the exciton in the state $|l\rangle$ and $\gamma_{l}$ is the recombination rate for this state. The latter depend on the delocalization of the exciton state ${ }^{3,5}$. Assuming that the electron and hole wave functions in a given dot are similar and that the excited states $|6\rangle$ and $|7\rangle$ are bright, one has for the states defined in Eqs. (12) and (13) $\gamma_{0,1}=\gamma_{0}\left(1 \pm \sin \theta_{01}\right), \gamma_{6,7}=\gamma_{0}\left(1 \pm \sin \theta_{67}\right)$ (the upper sign corresponds to the first of the two indices), and all the remaining decay rates equal to 0 . Here $\gamma_{0}$ is the decay rate of an exciton on a single quantum dots; we assume $\gamma_{0}=1 \mathrm{~ns}^{-1}$.

As an example, we recalculate the results presented in Fig. 6, now taking into account the radiative decay. The results are shown in Fig. 11] The effect at short times is very small. On the long time scales, however, the coherence does not stabilize at a constant value but decays due to radiative recombination of the exciton. As a result of this process, the maximum value of the spatial coherence is lower than in the idealized model discussed in this paper.
* Electronic address: Pawel.Karwat@pwr.edu.pl

1 M. Scheibner, T. Schmidt, L. Worschech, A. Forchel, G. Bacher, T. Passow, and D. Hommel, Nat. Phys. 3, 106 (2007).

2 Y. Mazur, V. Dorogan, E. Marega, D. Cesar, V. LopezRichard, G. Marques, Z. Zhuchenko, G. Tarasov, and G. Salamo, Nano. Res. Lett. 5, 991 (2010).

3 A. Sitek and P. Machnikowski, Phys. Rev. B 75, 035328 (2007).

4 T. Nakaoka, E. C. Clark, H. J. Krenner, M. Sabathil, M. Bichler, Y. Arakawa, G. Abstreiter, and J. J. Finley, Phys. Rev. B 74, 121305(R) (2006).

5 P. Karwat, A. Sitek, and P. Machnikowski, Phys. Rev. B 84, 195315 (2011).

${ }^{6}$ M. Gross and S. Haroche, Phys. Rep. 93, 301 (1982).

7 M. Kozub, Ł. Pawicki, and P. Machnikowski, Phys. Rev. B 86, 121305(R) (2012).

8 E. A. Zibik, T. Grange, B. A. Carpenter, N. E. Porter, R. Ferreira, G. Bastard, D. S. S. Winnerl, M. Helm, H. Y. Liu, M. S. Skolnick, and L. R. Wilson, Nature Materials 8, 803 (2009).

9 O. Verzelen, G. Bastard, and R. Ferreira, Phys. Rev. B 66, 081308(R) (2002).

10 B. Krummheuer, V. M. Axt, and T. Kuhn, Phys. Rev. B 65, 195313 (2002).

11 K. Gawarecki, M. Pochwała, A. Grodecka-Grad, and P. Machnikowski, Phys. Rev. B 81, 245312 (2010).

12 K. Gawarecki and P. Machnikowski, Phys. Rev. B 85, 041305(R) (2012).

13 K. C. Wijesundara, J. E. Rolon, S. E. Ulloa, A. S. Bracker,
D. Gammon, and E. A. Stinaff, Phys. Rev. B 84, 081404 (2011).

14 T. Nakaoka, J. Tatebayashi, Y. Arakawa, and T. Saito, J. Appl. Phys. 96, 150 (2004).

15 K. Müller, A. Bechtold, C. Ruppert, M. Zecherle, G. Reithmaier, M. Bichler, H. J. Krenner, G. Abstreiter, A. W. Holleitner, J. M. Villas-Boas, M. Betz, and J. J. Finley, Phys. Rev. Lett. 108, 197402 (2012).

16 A. O. Govorov, Phys. Rev. B 68, 075315 (2003).

17 E. Rozbicki and P. Machnikowski, Phys. Rev. Lett. 100, 027401 (2008).

18 B. D. Gerardot, S. Strauf, M. J. A. de Dood, A. M. Bychkov, A. Badolato, K. Hennessy, E. L. Hu, D. Bouwmeester, and P. M. Petroff, Phys. Rev. Lett. 95, 137403 (2005).

19 E. A. Muljarov, T. Takagahara, and R. Zimmermann, Phys. Rev. Lett. 95, 177405 (2005).

20 P. Machnikowski, Phys. Rev. Lett. 96, 140405 (2006).

21 Q. Xie, A. Madhukar, P. Chen, and N. P. Kobayashi, Phys. Rev. Lett. 75, 2542 (1995).

22 B. Szafran, T. Chwiej, F. M. Peeters, S. Bednarek, J. Adamowski, and B. Partoens, Phys. Rev. B 71, 205316 (2005).

23 B. Szafran, Acta Phys. Polon. A 114, 1013 (2008).

24 J. M. Daniels, P. Machnikowski, and T. Kuhn, Phys. Rev. B 88, 205307 (2013).

25 A. Grodecka, L. Jacak, P. Machnikowski, and K. Roszak, in Quantum Dots: Research Developments, edited by P. A. Ling (Nova Science, NY, 2005), p. 47, cond-mat/0404364. 26 H.-P. Breuer and F. Petruccione, The Theory of Open 
Quantum Systems (Oxford University Press, Oxford, 2002).

27 Z. J. Wu, K. D. Zhu, X. Z. Yuan, Y. W. Jiang, and H. Zheng, Phys. Rev. B 71, 205323 (2005).
28 J. Danckwerts, K. J. Ahn, J. Förstner, and A. Knorr, Phys. Rev. B 73, 165318 (2006). 